\title{
Present status of storage rings at HIRFL
}

\section{Youjin Yuan ${ }^{1}$}

Institute of modern physics, CAS

Lanzhou, China

E-mail: yuanyj@impcas.ac.cn

\section{Jiawen Xia}

Institute of modern physics, CAS

Lanzhou, China

E-mail: xiajw@impcas.ac.cn

Present status of storage rings CSRm and CSRe at Heavy Ion Research Facility at Lanzhou (HIRFL) of Institute of Modern Physics (IMP) is introduced. During 3 years operation and upgrading, the performance of CSR is getting ready for high level physics experiments including nuclear physics related to high precision mass measurement(IMS), hadron cancer therapy and atomic physics research. In addition, further enhancements of the facility are undergoing including Schottky mass spectrometer (SMS) and stochastic cooling in CSRe, upgrading of the injector will be introduced.

8th International Conference on Nuclear Physics at Storage Rings (Stori11)

Laboratori Nazionali di Frascati dell'INFN, Italy

October 9-14, 2011

\footnotetext{
$1 \quad$ Speaker
} 


\section{Introduction and Operation Status of HIRFL}

HIRFL is the major facility of national laboratory of heavy ion accelerators. It is founded in 1991 as one of the three national laboratories of China, focused on nuclear physics, atomic physics and heavy ion related application and cross-disciplinary. HIRFL-CSR as an upgrading project of HIRFL is constructed during 2000 to 2005, it's put into operation in 2008 after 2 years commissioning and testing. The layout of HIRFL is shown in Figure 1, the major parameters of HIRFL are listed in Table 1. [1,2,3,4]. The two cyclotrons SFC and SSC, built in 1963 and 1989 separately, are used as the injectors of CSR. In HIRFL-CSR project, CSRm is used as storage ring and synchrotron, RIBLL2 is used as fragment separator and CSRe is used as multifunction experimental ring.

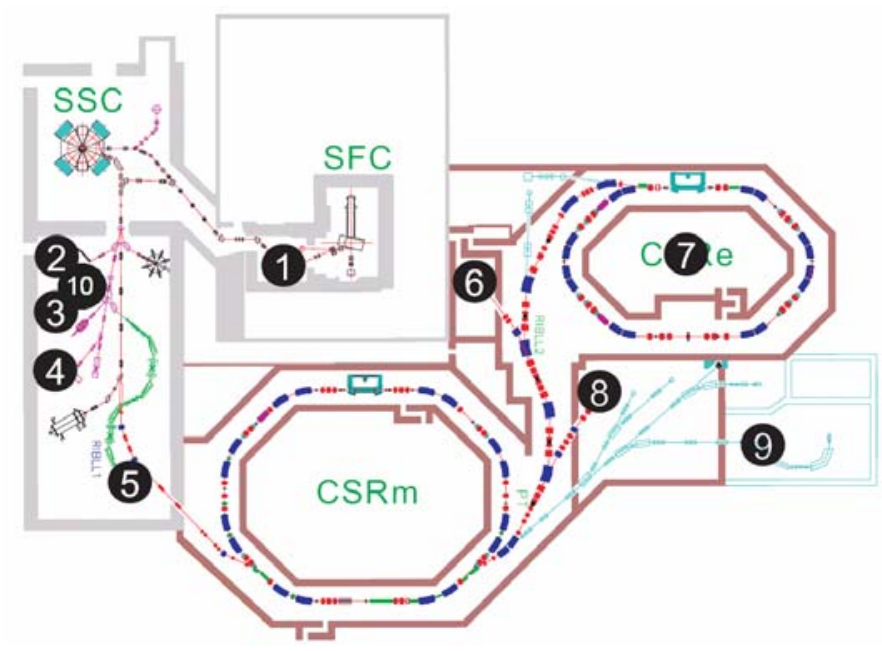

1. Low energy site in general

2. Atomic physics terminal

6. ETF-external target for nuclear physics, neutron wall

3. SHANS - Spectrometer for heavy atom and nuclear Structure

7. RIBLL2 and CSRe

Heavy ion irradiation

5. RIBLL $1-1^{\text {st }}$ RIB line

8. PISA from COSY

9. Tumour therapy

10. Micro-beam terminal

Figure 1: Distribution of experiment terminals at HIRFL

\begin{tabular}{|l|l|l|l|l|l|}
\hline Ion species & \multicolumn{2}{l|}{ Proton $\sim$ Uranium } & & CSRm & CSRe \\
\hline & & & Magnet rigidity & $0.7 \sim 11.5 \mathrm{Tm}$ & $0.6 \sim 8.9 \mathrm{Tm}$ \\
\hline & SFC & SSC & e-Cooler & $1-35 \mathrm{kV}$ & $50-300 \mathrm{kV}$ \\
\hline $\mathbf{k}$ & 69 & 450 & Vacuum & $<610^{-11} \mathrm{mbar}$ & $<6 \times 10^{-11} \mathrm{mbar}$ \\
\hline Magnet rigidity & $<1.2 \mathrm{Tm}$ & $<3.1 \mathrm{Tm}$ & RF cavity & $0.25 \sim 1.7 \mathrm{MHz} / 7 \mathrm{kV}$ & $0.5 \sim 2 \mathrm{MHz} / 2 \times 10 \mathrm{kV}$ \\
\hline Emittance & $\sim 24 \pi$ & $\sim 5 \pi$ & Extraction & Fast $/ \mathrm{Slow}(\mathrm{RF} \mathrm{KO})$ & - \\
\hline
\end{tabular}

Table 1: Major Parameters of HIRFL

Presently, there are about 10 major terminals around HIRFL, they are listed in Figure 1. In operation of recent years, experimental terminals around cyclotrons and the storage rings shared the beam time almost equally. As an example, the distribution of operation time of HIRFL in operation year 2010-2011 is shown in Table 2. 
HIRFL has provided beams for experiments of 19 chemical elements with different isotopes. Benefiting from superconductive ECR source SECRAL[5,6], metallic and rare gas isotopes are supplied with high charge states. The blocks of Figure 2 that coloured in yellow and red indicate the elements accelerated in recent years at HIRFL, the red ones indicate those accelerated by CSRm. The operation parameters of supplied beams are listed in Table 3 .

\begin{tabular}{|l|r|r|r|r|}
\hline \multirow{2}{*}{} & \multicolumn{2}{|c|}{ HIRFL } & \multicolumn{2}{c|}{ HIRFL-CSR } \\
\cline { 2 - 5 } & Time $(\mathrm{h})$ & Percentage & Time $(\mathrm{h})$ & Percentage \\
\hline Total operation time & 7824 & $100 \%$ & 4018 & $100 \%$ \\
\hline On-target beam time & 4809 & $61.5 \%$ & 2698 & $67.2 \%$ \\
\hline Beam preparation & 2609 & $33.3 \%$ & 1068 & $26.5 \%$ \\
\hline Hardware Failure & 406 & $5.2 \%$ & 252 & $6.3 \%$ \\
\hline
\end{tabular}

Table 2: Distribution of HIRFL operation time in operation year 2010-2011

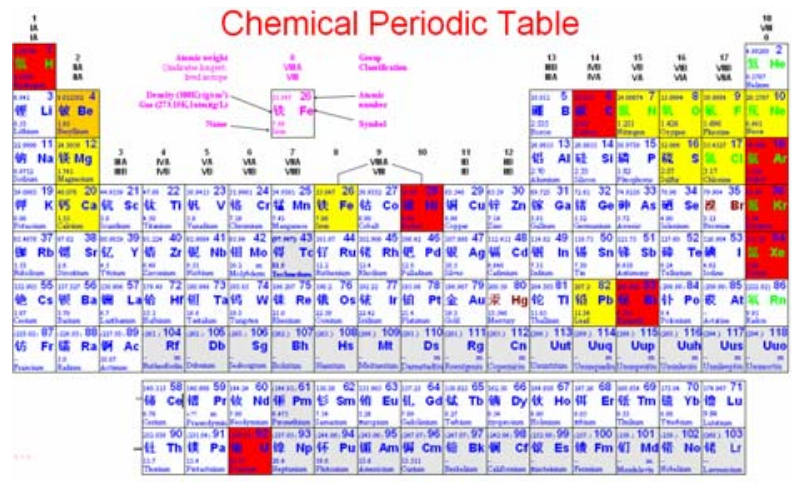

Figure 2: Accelerated elements at HIRFL

\begin{tabular}{|c|c|c|c|c|c|c|}
\hline Beam & $\begin{array}{l}\text { Inj- } \\
\text { ector }\end{array}$ & $\begin{array}{l}\text { Acc. } \\
\text { Sch. }\end{array}$ & $\begin{array}{l}\text { Energy } \\
(\mathrm{MeV} / \mathrm{u})\end{array}$ & $\begin{array}{l}\text { Int. } \\
\text { (ppp) }\end{array}$ & $\begin{array}{l}\text { Exp. } \\
\text { Terminal }\end{array}$ & \multirow{9}{*}{$\begin{array}{l}\text { MMI: multiple } \\
\text { multi-turn injection, } \\
\text { CEI: charge } \\
\text { exchange } \\
\text { IMS: isochronous } \\
\text { mass spectroscopy } \\
\text { of CSRe } \\
\text { CT: cancer therapy, } \\
\text { ETF: external target } \\
\text { facility, IT: internal } \\
\text { target }\end{array}$} \\
\hline $\mathbf{H}_{2}^{+}$ & SFC & MMI & $200 \sim 800$ & $2 \times 10^{8}$ & CSRm & \\
\hline${ }^{12} C^{6+}$ & SFC & CEI & $\begin{array}{l}150 \sim 600 / \\
600\end{array}$ & $\begin{array}{l}2 \times 10^{8} / \\
7 \times 10^{9}\end{array}$ & $\begin{array}{l}\mathrm{CT} / \mathrm{ETF} / \\
\mathrm{CSRe}\end{array}$ & \\
\hline${ }^{36} \mathrm{Ar}^{18+}$ & $\begin{array}{l}\text { SFC+ } \\
\text { SSC }\end{array}$ & MMI & $368 \sim 500$ & $4 \times 10^{8}$ & CSRe/IMS & \\
\hline${ }^{58} \mathrm{Ni}^{19+}$ & SFC & MMI & $197 \sim 235$ & $1 \times 10^{8}$ & CSRe/IMS/IT & \\
\hline${ }^{78} \mathrm{Kr}^{28+}$ & SFC & MMI & $300 \sim 500$ & $2 \times 10^{8}$ & CSRe/IMS & \\
\hline${ }^{129} \mathrm{Xe}^{27+}$ & SFC & MMI & $197 \sim 235$ & $1 \times 10^{8}$ & CSRe/IT & \\
\hline${ }^{209} \mathrm{Bi}^{36^{+}}$ & SFC & MMI & $197 \sim 235$ & $5 \times 10^{7}$ & CSRm & \\
\hline${ }^{238} \mathbf{U}^{32+}$ & SFC & MMI & $197 \sim 235$ & $1 \times 10^{8}$ & $\mathrm{CSRm}$ & \\
\hline
\end{tabular}

Table 3: Major parameters of CSR operation

\section{The Operation modes of storage rings and RIBLL2}

\subsection{Operation Modes of CSRm}

CSRm is the main synchrotron of CSR project. It's of 4-folder symmetry. Due to the lower limit of RF frequency and systematic magnet field error of measurement, the acceleration procedure is divided into two steps. At the first step, acceleration may use higher harmonic number; the working tunes can be corrected. 
With large acceptance, two injection and accumulation methods are realized [7]: one is the multiple multi-turn injection (MMI) with cooling, designed for general accumulation; the other is charge exchange injection(CEI) with cooling designed for light nuclei. The major lattice parameters of CSR are shown in Figure 3.

For accumulation and acceleration, CSRm works at tune values 3.63/2.62. The $1 / 3$ integer resonant in horizontal plane is designed for slow extraction [7]. For slow extraction at the energy flattop, the horizontal tune value is set to 3.663 , the chromaticity is corrected to zero in both plane and the dispersion function at first extraction inflector is set zero. The area of stable separatrix triangle for all momentum deviation $(<0.1 \%)$ is designed as $13 \pi \mathrm{mm}$.mrad. RF knock-out scheme is adopted to excite the slow extraction. With a scanned $(0.2 \%)$ frequency around $1.663 \mathrm{f}_{\mathrm{rev}}$, ions are extracted with amplitude selection. A feedback system is introduced to make smoothly extracted beam; a pair of fast quadrupoles is used to stabilize the horizontal tune value.

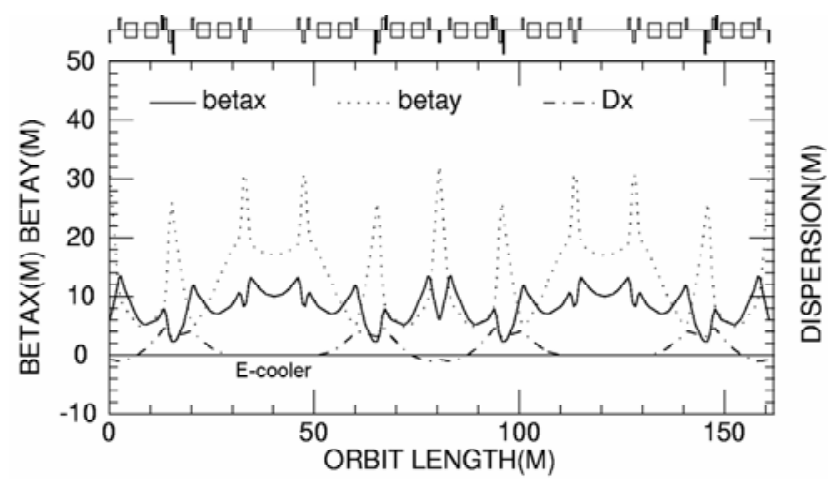

Figure 3: Lattice parameters of CSRm

\subsection{Operation Modes of CSRe}

CSRe is a multifunction experimental cooling storage ring, see Figure 4. There are three major operation modes available; they are normal, internal target and isochronous mass spectroscopy (IMS) mode.

The normal mode has a large momentum acceptance, $\Delta \mathrm{P} / \mathrm{P}=2.6 \%$. This mode is designed for general usage, it's good for electron cooling related experiments, for example: atomic physics and Schottky mass spectroscopy (SMS), some of the internal target experiments. The lattice parameters for normal mode are shown in Figure 5, it's almost symmetry.

The internal target mode is specially designed for experiments of internal target related experiments. The beam size at internal target site is minimized. The lattice parameters are shown in Figure 5. The acceptance of internal target mode is $A_{x, y}=150 / 75 \pi \mathrm{mm} . \mathrm{mrad}$, $\Delta \mathrm{P} / \mathrm{P}=2 \%$.

The IMS mode of CSRe[1] is designed for precise mass measurement of the short-lived RIB. With a small transition energy, $\gamma_{\mathrm{tr}}=1.395$, the revolution frequency of isochronous nuclei reached $10^{-5}$ within a momentum acceptance of $\Delta \mathrm{P} / \mathrm{P}<5 \times 10^{-3}$ (with TOF foil online). The lattice parameters are shown in Figure 6. With a half aperture injection kicker, in the IMS mode of CSRe, the closed orbit of fast injected nuclei is distorted, see Figure 6. The aperture of TOF carbon foil is $40 \mathrm{~mm}$, its thickness is $15 \mu \mathrm{g}$. The frame of carbon foil limits the acceptance. 
The dispersion at internal target is $\sim 15 \mathrm{~m}$, when two new TOF detectors are installed near this section, the acceptance will be further reduced. The closed orbits of these modes at cooler section are similar at injection time, as shown in Figure 6. Before electron cooling take effects the distorted orbits should be ramped to centre orbit to make both beam parallel.

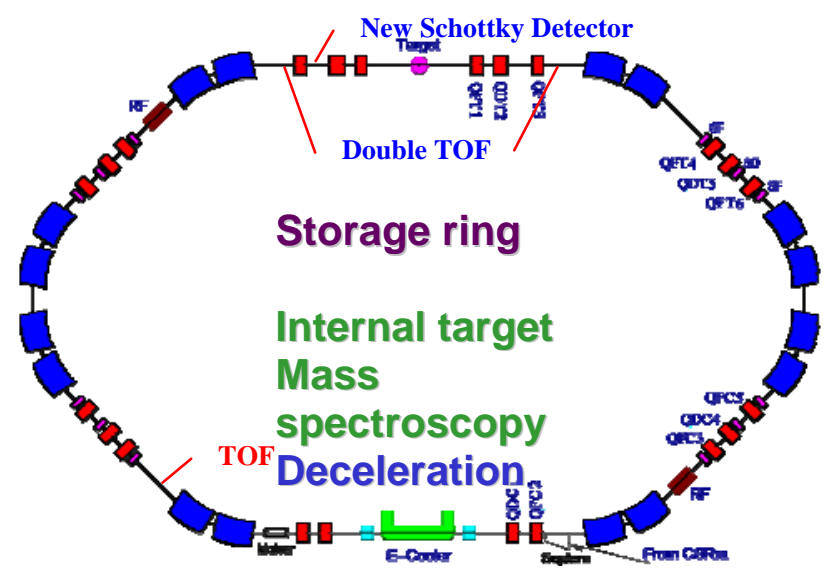

Figure 4: Layout of CSRe
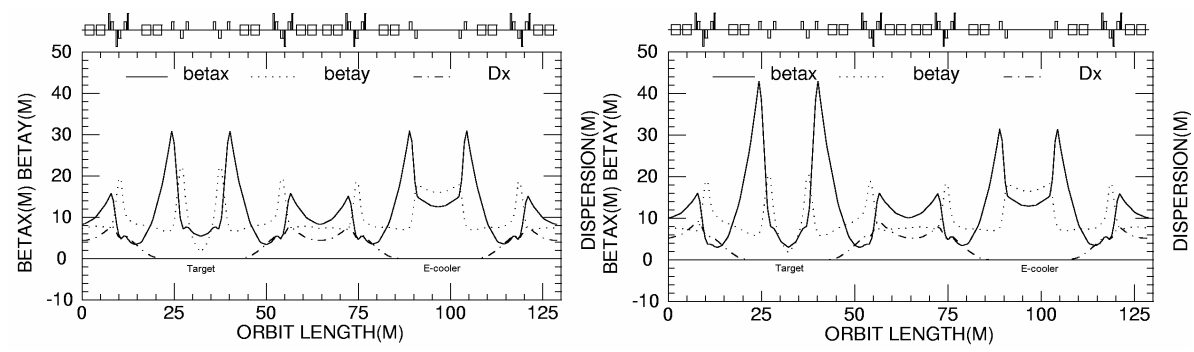

Figure 5: Lattice parameters of CSRe normal mode and internal target mode(right)
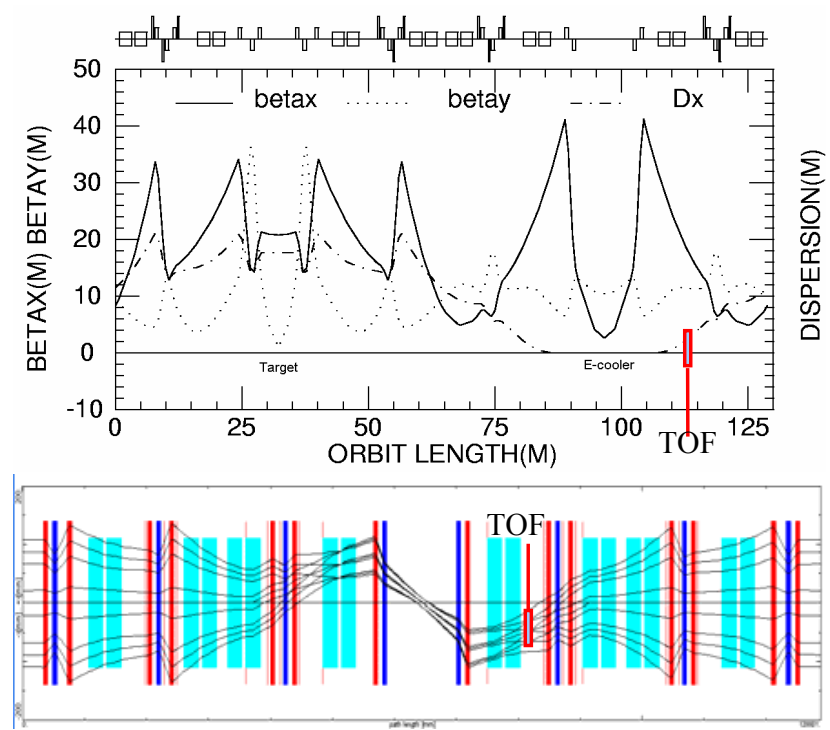

Figure 6: Lattice parameters and beam envelopes of stored ions with different momentum at IMS mode. 


\subsection{0peration Modes of RIBLL2}

The RIBLL2 is designed as a double achromatic fragment separation beam line [9]. The layout and beam envelopes are shown in Figure 7. The momentum acceptance is $\Delta \mathrm{P} / \mathrm{P}= \pm 1 \%$, with emittance of $25 \pi \mathrm{mm}$.mrad in both $\mathrm{x}$ and $\mathrm{y}$ plane. The resolution power is 1200 .
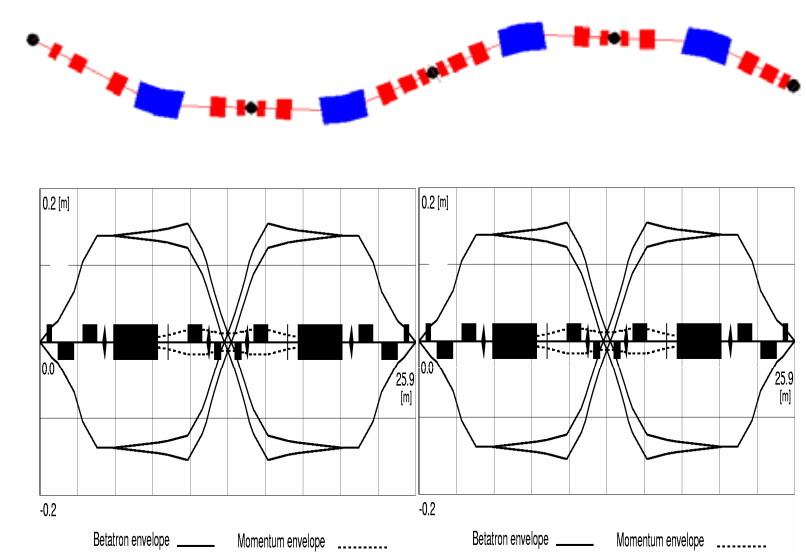

Figure 7: Layout and envelopes of RIBLL2.

RIBLL2 is used as beam transfer line to feed beam from CSRm to CSRe at present, further study with sufficient detectors and monitors is necessary before it is fitted to RIB separation.

\section{Experiments at storage rings}

Experiments at storage rings are focused on precise atom mass measurements, tumour therapy studies and ion irradiation physics. Some other experiments including atomic physics, nuclear physics and high energy physics experiments are in development and testing stage.

\subsection{CSRe IMS Experiments [10,11,12]}

After the testing experiment in 2008, several runs of experiments using IMS at CSRe are done with good results $[10,11,12]$. The primary beam of ${ }^{36} \mathrm{Ar},{ }^{78} \mathrm{Kr}$ and ${ }^{58} \mathrm{Ni}$ is supplied.

The long term magnet field drift of CSRe [1] is improved recently from $\sim 10^{-3}$ to $10^{-5}$. After minor correction of sextupoles, the frequency dispersion of primary beam at isochronous energy is better than $10^{-5}$ within the momentum acceptance.

As shown in Figure 4, a pair of new TOF detectors will be installed at the straight section of CSRe internal target. With double TOF, one can measure the energy of each nuclei turn by turn precisely, the systematic error of mass measurement will be further reduced.

The new resonant cavity Schottky pickup is also installed at the section, it will be used in experiments of SMS to detector revolution frequency of each single nuclei. The stochastic cooling at CSRe is under construction. With the Schottky pickup, stochastic cooling and electron cooling, the SMS related physics experiments will be extremely extended.

\subsection{Progress of Cancer Therapy Study}

The tumour treatment study at cancer therapy terminal is processing smoothly during last 3 years. Up to May 2011, 45 patients including are treated. The beam quality and machine 
stability are improved steadily. Fast energy stepping from pulse to pulse, 3D raster scanning and single charge exchange injection are realized, they are the key techniques of HITFiL - a new project of heavy ion treatment facility at Lanzhou, which started in 2010.

\subsection{Atomic Physics Experiment}

Experiment setups for atomic physics at e-cooler section of CSRm and CSRe, internal target section of CSRe are ready. Several testing experiments are done with good results. Laser cooling of high energy beam at CSRe will be prepared.

\subsection{ETF Experiment}

For testing of the detector matrix at stationary target area, $\mathrm{C}^{6+}-600 \mathrm{MeV} / \mathrm{u}$ was slow extracted and transported through Beryllium foil to a stationary target outside the vacuum chamber. The test experiment observed and distinguished mesons for the first time in IMP. The stability of beam position on the target should be improved to increase the resolution of spectrum.

\section{Developments of HIRFL in near-future}

Urgent requirements of developing at HIRFL are listed as following:

1. High beam intensity of heavier nuclei from SSC, which will be further stripped to higher charge states and accelerated to higher energy in CSRm.

2. More on-target beam time to match the increasing needs.

3. The stability and efficiency of operation, easy commissioning.

4. Stochastic cooling at CSRe.

5. Deceleration of beam at CSRe.

A series of development will be done at HIRFL recently to further enhance the machine:

1. A new heavy ion Linac will be built as a new injector of SSC, focusing on the high intensity of heavy ion beam. As an alternation, an intense heavy ion Linac as a new injector for CSRm will be a better choice.

2. Study of efficiency improvement of SSC.

3. Systematic upgrade of control, diagnosis, power supply and supporting systems to stabilize the operation.

4. Time sharing of beam from injectors will contributes to the total beam on-target time.

5. Stochastic cooling will be built at CSRe. Combined with electron cooling at CSRe, experiments related to SMS will start.

\section{References}

[1] Y.J. Yuan, H.W. Zhao, J.W. Xia et al, HIRFL-CSR facility status and development, in proceedings of Cyclotrons and Their Applications 2010.

[2] Y.J. Yuan, H.W. Zhao, J.W. Xia et al, Status of HIRFL-CSR Project, in proceedings of COOL 2009, 31 Aug - 4 Sep. 2009, Lanzhou, China. 
[3] W. L. Zhan, H. S. Xu, G. Q. Xiao etc., Progress in HIRFL-CSR, Nuclear Physics A, 834 (2010) 694c-700c, March 2010. NN2009, Beijing, China, 16-21 August 2009.

[4] W.L. Zhan, J.W. Xia, H.W. Zhao, et al, HIRFL today, Nuclear Physics A, 805: 533C-540C, June 2008, 23rd International Nuclear Physics Conference, Jun 03-08, 2007, Tokyo, Japan.

[5] Zhao HW, et al, New development of advanced superconducting electron cyclotron resonance ion source SECRAL. Rev. Sci. Instrum. 2010 Feb; 81(2):02A202.

[6] H. W. Zhao, et al, An advanced superconducting ECR ion source SECRAL at IMP: first results and operation at $18 \mathrm{GHz}$, Cyclotrons and Their Applications 2007.

[7] Y.J. Yuan, Injection and slow extraction for carbon ion therapy at IMP,NIRS-IMP Joint Symposium on Carbon Ion Therapy, August 2009, Lanzhou, China.

[8] M. Hausmann, F. Attallah, K. Beckert et al. First isochronous mass spectrometry at the experimental storage ring ESR. Nucl. Instrum. Methods Phys. Res. A446, 569 (2000).

[9] Song M.T. el al, High Energy Physics and Nuclear Physics, 25(5), p443-447(2001), in Chinese.

[10] Tu Xiao-Lin, Xu Hu-Shan, Xia Jia-Wen, et al, A pilot experiment for mass measurement at CSRe, Chinese Physics C, 33(7): 516-520, July 2009.

[11]H.S. Xu, X.L. Tu, Y.J. Yuan, et al, First mass measurement of short-lived nuclides at HIRFLCSR, Chinese Science Bulletin, 54 (24): 4749-4752, Dec 2009.

[12]X. L. Tu, H. S. Xu, M. Wang et al. Direct Mass Measurements of Short-Lived A=2Z-1 Nuclides 63Ge, 65As, 67Se, and 71Kr and Their Impact on Nucleosynthesis in the rp Process. Phys. Rev. Lett. 106, 112501 (2011).

[13]X.D. Yang, V.V. Parkhomchuk et al, Proceedings of COOL 2007, Bad Kreuznach, Germany, pp.59-63.

[14]L.J. Meng, X. Ma, H.P. Liu et al, The first test experiment performed at the electron cooler of storage rings in Lanzhou, (HCI 2008), IOP Publishing, Journal of Physics: Conference Series 163 (2009), doi:10.1088/1742-6596/163/1/012031.

[15]X.D. Yang, L.J. Mao, G.H. Li, et al, Commissioning of electron cooling in CSRe, in proceedings of COOL 2009, 31 Aug - 4 Sep. Lanzhou, China. 